\title{
La Swiss Medical Student Association SwiMSA
}

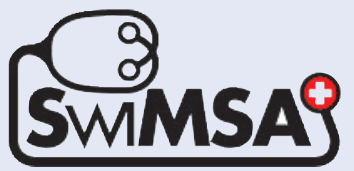

\section{Martin Krähenbühl}

Président de la SwiMSA et étudiant en médecine de $4^{\mathrm{e}}$ année
Correspondance: SwiMSA

Swiss Medical Student Association c/o Fachschaft Medizin Bern

Postfach 28

CH-3010 Berne

office@swimsa.ch

www.swimsa.ch
La Swiss Medical Student Association (SwiMSA) est l'association faîtière de toutes les organisations d'étudiants en médecine de Suisse. Elle défend leurs intérêts et soutient des projets dans lesquels les étudiants en médecine s'engagent durant et après leurs études. Elle assume leur défense sur le plan national, dans les universités et les associations professionnelles, et sur le plan international, au sein de la «Federation of Medical Students Associations» (IFMSA). Sa fonction de plate-forme permet aux associations membres d'utiliser les synergies et de se présenter ensemble aux étudiants. La SwiMSA facilite la mise en œuvre de nouvelles idées et la réalisation de projets.

\section{De «cliniciens» à «medical students»}

Les racines de la SwiMSA remontent à 1917, lorsqu'une première association d'étudiants en médecine vit le jour sous le nom d'Association des cliniciens de Suisse. Celle-ci eut l'honneur de participer en 1951 à la fondation de l'organisation «International Federation of Medical Students Associations», présente aujourd'hui sur toute la planète.

L'actuelle SwiMSA résulte du regroupement, en 2006, de plusieurs associations d'étudiants très diverses. Notre membre le plus important du point de vue numérique est la SwiMSA-Exchanges (autrefois IFMSA-Switzerland) qui organise des échanges de postes de stage pratique d'un mois dans le monde entier en collaboration avec l'IFMSA. Les organisations spécialisées défendent les intérêts des étudiants, notamment dans le

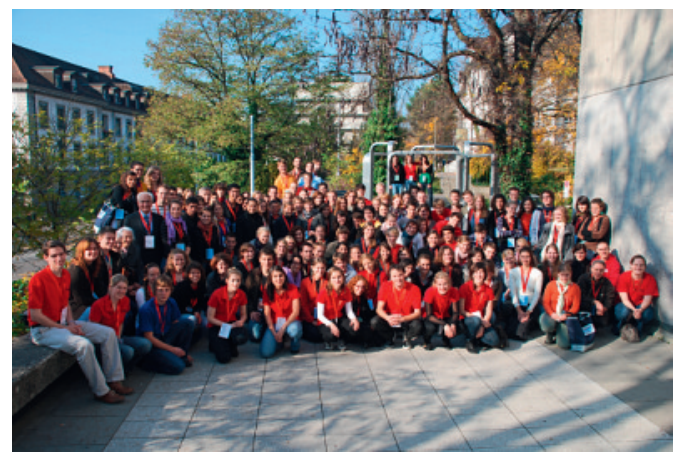

domaine de la politique universitaire. Notre association membre «GRUHU» soutient les étudiants zurichois dans des projets à l'étranger pour l'année à option et l'association «Achtung Liebe» donne des cours d'éducation sexuelle dans les écoles. Le PSR Suisse (Physicians for Social Responsibility) qui a rejoint la SwiMSA en 2008 est encore un petit groupe; son fer de lance est la lutte contre la menace nucléaire et la promotion des énergies alternatives.

\section{Les Conventions: lieux d'échanges et de mise au point de projets}

La SwiMSA, qui s'est fortement développée en peu de temps, a aussi traversé des crises. Grâce à l'engagement infatigable des chefs de domaine, elle a pu néanmoins enregistrer des succès remarquables. Les Conventions nationales, organisées chaque semestre, revêtent une grande importance. Ici, nos associations membres ont l'occasion unique de se présenter aux étudiants de toute la Suisse. Ici, les étudiants entrent en contact avec la SwiMSA et reçoivent des informations détaillées sur ses projets. Ici, les membres des associations relatent leurs expériences et participent au développement de nouveaux projets. La dernière Convention en particulier, tenue à Zürich, a été une manifestation couronnée de succès; elle a accueilli plus de 150 participants satisfaits ainsi que des hôtes représentant le Parlement et la politique de défense professionnelle des médecins.

\section{Voix audible des étudiants en médecine}

L'année dernière, la collaboration des organisations spécialisées d'étudiants a été renforcée et rendue plus efficace dans le domaine de la politique universitaire. Nous nous sommes rapprochés du but d'être un jour un partenaire à prendre au sérieux pour les décanats. En outre, nous avons mis sur pied le journal Med!campus destiné à informer les étudiants en médecine sur la SwiMSA, les associations membres, les études et les thèmes actuels concernant la médecine de façon générale. La deuxième édition de Med!campus va bientôt paraître. 